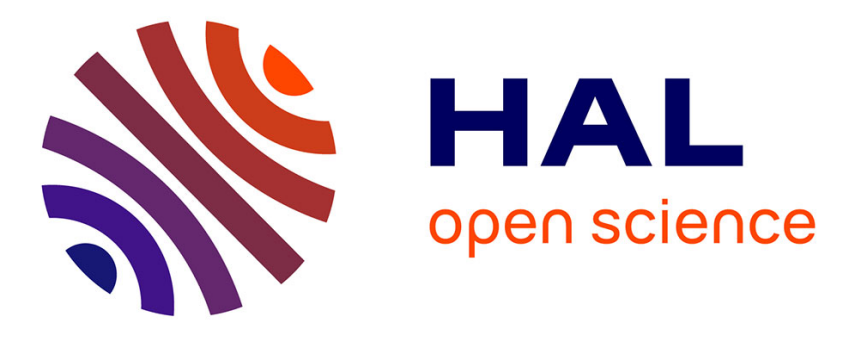

\title{
A vision-based path planner/follower for an assistive robotics project
}

Andrea Cherubini, Giuseppe Oriolo, Francesco Macri, Fabio Aloise, Febo

Cincotti, Donatella Mattia

\section{- To cite this version:}

Andrea Cherubini, Giuseppe Oriolo, Francesco Macri, Fabio Aloise, Febo Cincotti, et al.. A visionbased path planner/follower for an assistive robotics project. 1st International Workshop on Robot Vision, VISAPP 2007, 2007, Barcelone, Spain. hal-01247256

\section{HAL Id: hal-01247256 \\ https://hal.science/hal-01247256}

Submitted on 21 Dec 2015

HAL is a multi-disciplinary open access archive for the deposit and dissemination of scientific research documents, whether they are published or not. The documents may come from teaching and research institutions in France or abroad, or from public or private research centers.
L'archive ouverte pluridisciplinaire HAL, est destinée au dépôt et à la diffusion de documents scientifiques de niveau recherche, publiés ou non, émanant des établissements d'enseignement et de recherche français ou étrangers, des laboratoires publics ou privés. 


\title{
A vision-based path planner/follower for an assistive robotics project ${ }^{\star}$
}

\author{
Andrea Cherubini ${ }^{1}$, Giuseppe Oriolo ${ }^{1}$, Francesco Macrì ${ }^{1}$, \\ Fabio Aloise $^{2}$, Febo Cincotti ${ }^{2}$, and Donatella Mattia ${ }^{2}$ \\ ${ }^{1}$ Dipartimento di Informatica e Sistemistica \\ Università di Roma "La Sapienza" \\ Via Eudossiana 18, 00184 Roma, Italy \\ \{cherubini, oriolo\}@dis.uniromal.it \\ ${ }^{2}$ Fondazione Santa Lucia IRCCS \\ Via Ardeatina 306, 00179 Roma, Italy \\ ff.cincotti,d.mattia\}@hsantalucia.it
}

\begin{abstract}
Assistive technology is an emerging area where robots can be used to help individuals with motor disabilities achieve independence in daily living activities. Mobile robots should be able to autonomously and safely move in the environment (e.g. the user apartment), by accurately solving the self-localization problem and planning efficient paths to the target destination specified by the user. This paper presents a vision-based navigation scheme designed for Sony AIBO, in ASPICE, an assistive robotics project. The navigation scheme is map-based: visual landmarks (white lines and coded squares) are placed in the environment, and the robot utilizes visual data to follow the paths composed by these landmarks, and travel to the required destinations. Performance of this vision-based scheme is shown by experiments and comparison with two previously existing ASPICE navigation modes. Finally, the system is clinically validated, in order to obtain a definitive assessment through patient feedback.
\end{abstract}

\section{Introduction}

The development of service robots for healthcare and assistance to elderly or disabled persons is an active area of research. A typical use of robots in this context is directed to partial recovery of the patient mobility. Semi-autonomous navigation systems for wheelchairs are an example. In these applications, mobile robots must acquire a high degree of autonomous operation, which calls for accurate and efficient position determination and verification for safe navigation in the environment. In many recent works, this has been done by processing information from the robot vision sensors [1].

Implicitly or explicitly, it is imperative for a vision system meant for navigation to incorporate within it some knowledge of the environment. In particular, map-based

\footnotetext{
${ }^{\star}$ This work has been partially supported by the Italian Telethon Grant GUP03562. This manuscript describes variations of a work submitted at the IEEE International Conference on Robotics and Automation 2007: the main contribution concerns the ASPICE vision-based navigation mode - vision related topics have been extended in this version.
} 
robot navigation systems can be based on user-created geometric models within the environment [2]. The vision system will search and identify some known landmarks and use them, along with a provided map, to estimate the robot position (self-localization) by matching the observation (image) against the expectation (landmark descriptions). Visual navigation that uses patterns based on an identification code, has been exploited in [3]. More recent works include a guidance system for the visually impaired, based on visual cues on the ceiling [4], and a localisation system for autonomous wheelchairs based on light-emitting markers on the floor [5].

In this paper, we present a vision-based path planner/follower designed for an indoor structured environment. The system enables the robot to travel to a target destination, by extracting, visually tracking, and following, known artificial paths. This robot navigation system has been utilized within the ASPICE (Assistive System for Patient's Increase of Communication, ambient control and mobility in absence of muscular Effort) project [6]. The ASPICE system enables a disabled user to remotely control homeinstalled appliances, including the Sony AIBO, a commercial mobile robot.

The paper is organized as follows. In Sect. 2, the main features of the AIBO robot are described. Section 3 presents the vision primitives developed for the robot framework. The vision-based navigation scheme that we designed and implemented, is outlined in Section 4. Experiments are reported in Section 5. Other issues not covered in the previous sections are mentioned in the conclusion.

\section{The Robot Platform: AIBO}

The platform used in this work is a quadruped robot, Sony AIBO ERS-7, pictured in Fig. 1. The robot is equipped with 20 actuated joints, a CMOS camera, two distance sensors (on the head and on the chest), and other equipment not used in this work. A wireless card enables remote control. AIBO's real-time operating system APERIOS runs a specialized layer called OPEN-R, a cross-development environment based on $\mathrm{C}++$. The robot behavior is programmed by loading executable and configuration files on a memory stick which is read by the on-board processor. From a kinematic viewpoint, AIBO can be considered as an omnidirectional robot, i.e., three velocities (forward $v_{x}$, lateral $v_{y}$, and angular $v_{\theta}$ around the robot center) can be independently specified (Fig. 2). In spite of these features, AIBO presents many limitations. The most severe are:

- the closed hardware prevents the addition of sensors and/or actuators;

- since Sony does not release the code of its driver, we had to realize from scratch an ad hoc driver for this work;

- vibrational and slipping effects during the quadruped gait cycle make odometric reconstruction very inaccurate in the long run;

- the variable attitude of AIBO during its gait precludes the use of an external sensory system (e.g., based on infrared triangulation with a detector placed on the robot) for solving the localization problem. 

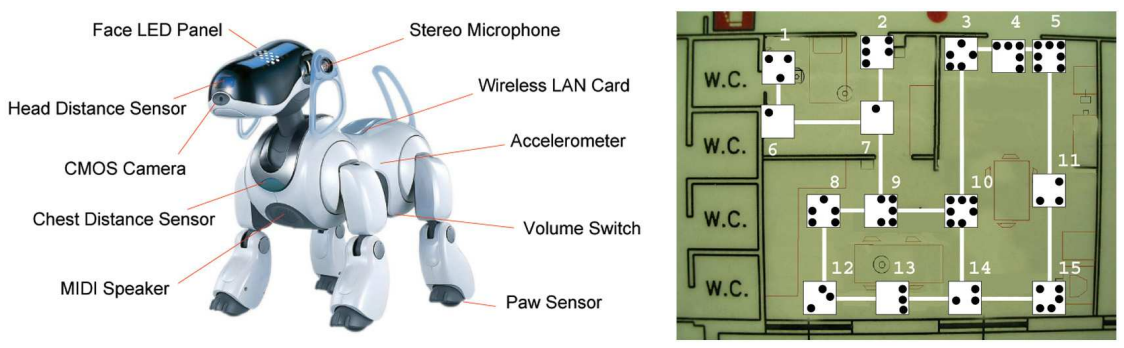

Fig. 1: ASPICE features: the robot Sony AIBO ERS-7(left), and the roadmap used for visionbased navigation, with the ID labels of each coded square (right).

\section{Vision primitives}

In order to extract and track the artificial visual landmarks (VL) on the navigation path, specific vision primitives have been developed and integrated in the driver framework. Let us define the three reference frames which will be used (shown in Fig. 2):

- the robot frame with origin fixed at the robot center projection on the ground, $x$ axis in the forward direction, $y$ axis pointing left, and $z$ axis vertical;

- the camera frame with origin fixed in the camera center, horizontal ${ }^{c} x$ axis pointing right, ${ }^{c} y$ axis pointing downward, and ${ }^{c} z$ axis pointing forward;

- the image frame with origin fixed at the top left corner of the image, horizontal ${ }^{i} x$ axis pointing right, and ${ }^{i} y$ axis pointing downward.

The VLs that we use are straight white lines (SWL) and coded squares (CS) placed on the floor. Thus, a straight white line extractor (SWLE) and a coded square extractor (CSE) have been developed. Moreover, the VLs should be located in sequential scenes. This is accomplished by a visual landmark tracker (VLT).

\subsection{Straight white line extractor}

A requirement of the robot driver is straight white line extraction. In order to be independent from color classification, the SWLs are detected by search only on the luminance signal $I\left({ }^{i} x,{ }^{i} y\right)$. Thus, line edges are searched at pixels with a strong variation of luminance with respect to that of adjacent pixels. For each pixel located in $p=\left[{ }^{i} x{ }^{i} y\right]^{T}$ the gradient of luminance $\nabla I(p)$ is computed, using the Roberts operator [7] as in [8]:

$$
\begin{array}{ll}
s(p)=I\left({ }^{i} x+1,{ }^{i} y+1\right) & t(p)=I\left({ }^{i} x+1,{ }^{i} x,{ }^{i} y\right)-I\left({ }^{i} x,{ }^{i} y+1\right) \\
|\nabla I(p)|=\sqrt{s(p)^{2}+t(p)^{2}} & \angle \nabla I(p)=\operatorname{ATAN} 2(s(p), t(p))
\end{array}
$$

where $|\nabla I(p)|$ is the magnitude and $\angle \nabla I(p) \in(-\pi, \pi]$ is the direction of the pixel luminance gradient (with respect to line ${ }^{i} y=-{ }^{i} x$ and positive CW, see Fig. 2). Edges are then detected by applying a threshold test to $|\nabla I(p)|$. We use a threshold dependent on the mean value of $|\nabla I(p)|$ on the given image to derive the set of image edge pixels $P_{e}$ 


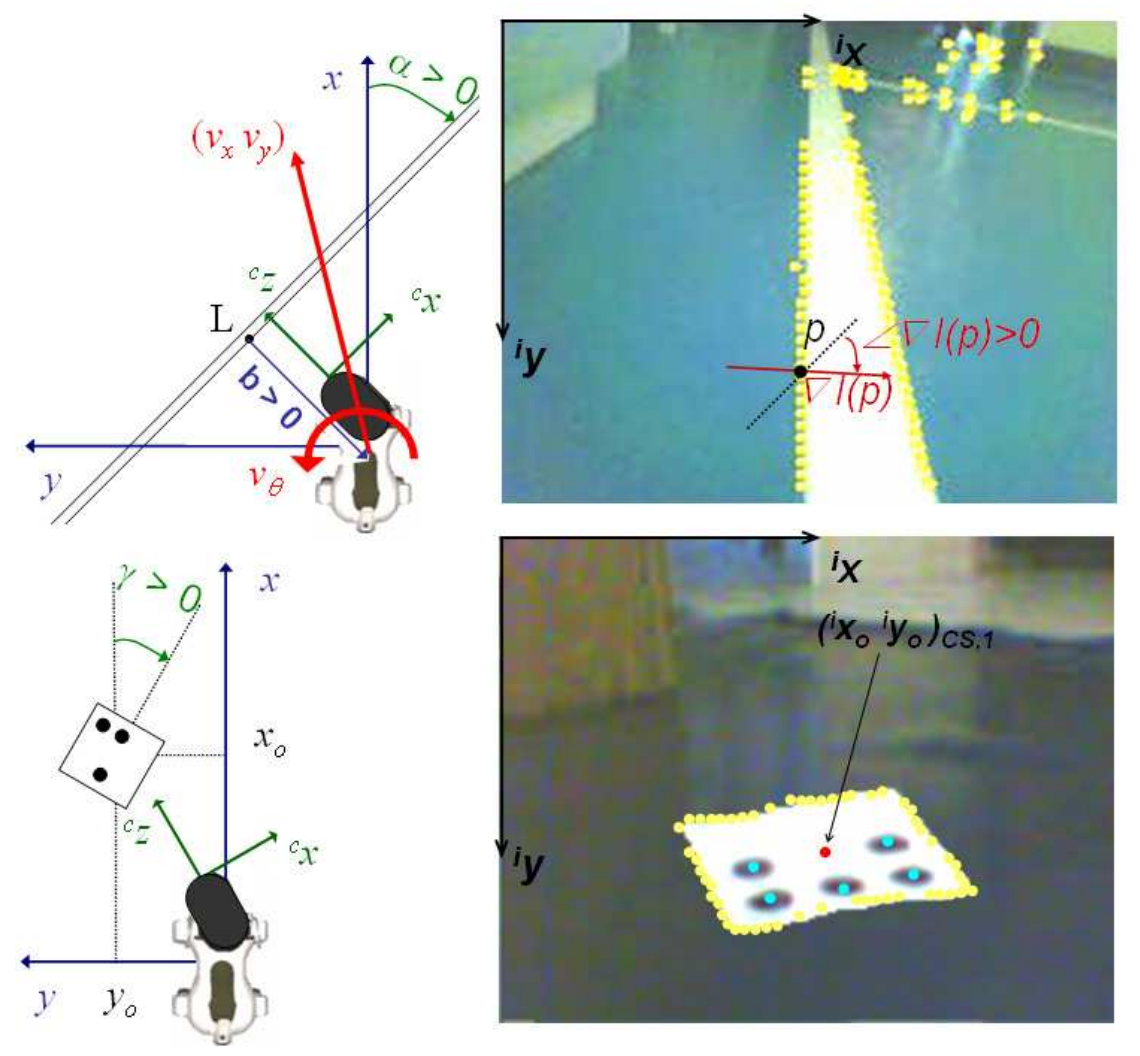

Fig. 2: Relevant variables utilized in: extraction and tracking of straight white lines (above), and extraction and tracking of coded squares (below).

(marked in yellow in Fig. 2). Adaptive thresholding makes the edge detection algorithm more robust to varying light conditions, as compared to similar works implemented in environments where light conditions had to be controlled. Afterwards, by comparing (with another threshold test) the luminance gradient directions of "near" edge pixels, pixels belonging to straight lines are identified and grouped in subsets. Indicating with $N_{S W L}$ the total number of straight white lines extracted on an image, the line pixel subsets are noted: $P_{S W L, j}\left(j=1 \ldots N_{S W L}\right)$. Each $P_{S W L, j}$ defines a line detected on the image, and must contain at least $n_{S W L, \text { min }}$ pixels.

We tested other edge detection algorithms (Sobel, Susan), but the Roberts operator showed better results, although the aforementioned algorithms are more efficient for color space based extraction [9]. Besides, due to its low computation time, this line extraction method was preferred to the Hough Transform technique, which we also experimented. In conclusion, the SWLE algorithm returns the coordinates of the pixels belonging to the $N_{S W L}$ lines extracted on the image frame (Fig. 2):

$$
\left[{ }^{i} x_{r}{ }^{i} y_{r}\right]_{S W L, j}^{T} \in P_{S W L, j} \quad r=1, \ldots, n_{j} \quad j=1, \ldots, N_{S W L}
$$




\subsection{Coded square extractor}

Along with the SWLs, we have used as visual landmarks a set of white coded squares laid on the ground. The identity and orientation of each square is uniquely identified through a black dots code, as in [2]. The choice of binary coding, i.e., black and white, is aimed at using luminance variation, instead of color classification, for extracting the CS characteristics. We arranged from 1 to 7 black dots on the squares border, in order to generate configurations which uniquely define the landmark identity (defined by its label: $I D$ ) and orientation. The 15 landmarks which we used can be seen in Fig. 1.

In practice, edges of squares are searched within the set of edge pixels $P_{e}$ derived as in the SWLE. The robot frame coordinates of all edge pixels are derived from their image frame coordinates, with the projection which will be presented below. Then, the projected edges are fitted with a square mask with the same dimensions as the CS, in order to detect the pixels belonging to the square perimeter. These define a subset of $P_{e}$ for each square (see Fig. 2). Indicating with $N_{C S}$ the total number of coded squares extracted on the image, the subsets of perimeter pixels of each square $l$ are noted: $P_{C S, l}\left(l=1 \ldots N_{C S}\right)$. Each $P_{C S, l}$ defines a coded square detected on the image, and must contain at least $n_{C S, \min }$ pixels. Afterwards, the image is scanned along the segments (scanlines) leading from the center to the perimeter of the CS, and pixels on such segments are classified by using a binary segmentation which uses the mean value of $I$ on the image as threshold. Corke [10] showed how binary segmentation is affected by noise, threshold selection and edge gradient. However, in this application, the choice of binary coding and the use of binary segmentation only in a small image window, and with adaptive thresholding, reduces these problems. Finally, black dots are extracted by associating a sufficient number of near black pixels found on the scanlines.

In conclusion, the CSE returns, for each of the $N_{C S}$ detected coded squares: the image coordinates of the square center $o$ and of the centers of the $n_{\text {dots }}$ black dots (respectively marked in red, and in cyan in Fig. 2):

$$
\begin{aligned}
& {\left[{ }^{i} x_{o}{ }^{i} y_{o}\right]_{C S, l}^{T} \quad\left[{ }^{i} x_{m}{ }^{i} y_{m}\right]_{C S, l}^{T}} \\
& l=1, \ldots, N_{C S} \\
& m=1, \ldots, n_{\text {dots }} \quad n_{\text {dots }}=1, \ldots, 7
\end{aligned}
$$

\subsection{Visual landmark tracker}

The SWLE and CSE only take into account information from the current image, and give no long-term knowledge. Thus, consistent landmarks must be obtained by comparing the extracted landmarks over consecutive images. This is done by projecting the characteristic points of each extracted visual landmark VL from the image frame

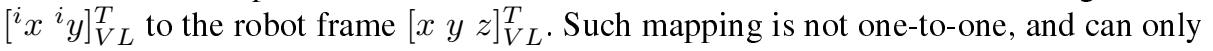
determine the projecting ray of the point. However, in our application, since all the VLs are on the ground plane, the problem can be solved in closed form.

In fact, given the intrinsic parameters of the camera, the projecting ray in camera frame coordinates corresponding to each $\left[{ }^{i} x{ }^{i} y\right]_{V L}^{T}$ can be derived [10]. Besides, the homogeneous transformation matrix representing the camera frame pose with respect to the robot frame can be computed through the robot joint positions by using the Denavit and Hartenberg method as in [8]. This matrix can be used, along with the projecting ray 
expression in camera frame coordinates, and with the ground plane geometric constraint $z_{V L}=0$, to derive $\left[\begin{array}{lll}x & y & 0\end{array}\right]_{V L}^{T}$ in the robot frame.

The robot frame coordinates of SWL points $\left[\begin{array}{lll}x_{r} & y_{r} & 0\end{array}\right]_{S W L, j}^{T}$ are then processed with a least square error algorithm to identify the parameters $[b \alpha]_{S W L, j}^{T}$ of each line: $b$ is the signed distance between the nearest line point $L$ and the robot (positive for $y_{L}>0$ ), and $\alpha \in\left(-\frac{\pi}{2}, \frac{\pi}{2}\right]$ denotes the orientation offset. The CS characteristic points $\left[\begin{array}{ll}x_{o} & y_{o}\end{array}\right]_{C S, l}^{T}$

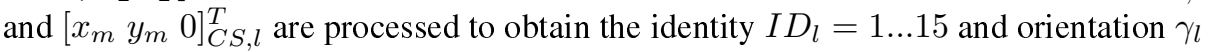
of each square. VLs extracted and projected at previous frames are displaced according to the robot measured motion and compared with the current landmarks for checking consistency and filtering out false positives. The algorithm returns the characteristics of the VLs, validated in a sufficient number of consecutive frames (Fig. 2):

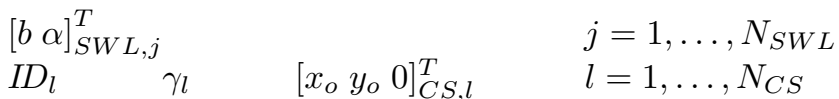

\section{The vision-based navigation scheme}

The vision-based navigation (VBN) scheme that we developed relies on a physical roadmap (Fig. 1) connecting all relevant destinations in the experimental arena, and on a visual servoing scheme. In practice, the robot plans and follows the path to destination according to visual data. Since the VLT returns the position of the visual landmarks relative to the robot, position-based visual servo control turns out to offer a better solution than image-based servoing [10]. The roadmap is formed by streets and crossings, all realized in white adesive tape and laid on the ground. The vision primitives are used to identify streets (i.e., straight white lines) and crossings (i.e., coded squares). The robot autonomous behavior is represented by a Petri Nets based framework which has been successfully deployed on the AIBO Platform in the Robocup field [11]. The VBN Plan uses the following actions: Seek nearest landmark, Approach nearest landmark, Follow street, Plan path to destination. Note that at all times during the actions, the vision primitives are also executed for searching and updating perceived data, and the robot keeps the nearest seen landmark centered in the image plane, by solving the inverse kinematics problem for the head joints. The VBN Plan repeats the actions until the destination is reached. Transitions that start or terminate the actions represent events (e.g., Street seen, or Crossing near) which are triggered by conditions on sensed information (e.g., distance from a line). In the rest of this section, the four actions are briefly described.

Seek nearest landmark AIBO seeks VLs by exploring the environment, while avoiding collisions. The robot alternates predefined forward and rotation steps.

Approach nearest landmark When it perceives a landmark (preferably a crossing) with the SWLE or CSE, and tracks it with the VLT, the robot approaches it, in order to get a better perception, which can be useful for localization purposes. It is a posture stabilization task with reference configuration defined by the position and orientation of the landmark. The walk that drives the robot implements a proportional closed-loop control strategy for reducing the robot relative distance and orientation with respect to 
the landmark. This is done by setting:

$$
\begin{aligned}
& v_{x}=\kappa_{\mathrm{T}} x_{V L} \\
& v_{y}=\kappa_{\mathrm{T}} y_{V L} \\
& v_{\theta}=-\kappa_{\mathrm{R}} \theta_{V L}
\end{aligned}
$$

In the case of SWL approaching, $[x y \theta]_{V L}^{T}=[b \sin \alpha b \cos \alpha-\alpha]^{T}$. Similarly, for CS, $\left[\begin{array}{lll}x & y & \theta\end{array}\right]_{V L}^{T}=\left[\begin{array}{ll}x_{o} & y_{o}-\gamma\end{array}\right]^{T}$. In both cases, $\kappa_{\mathrm{T}}$ and $\kappa_{\mathrm{R}}$ are positive given gains.

Follow street When the robot is sufficiently near to a street but has not yet detected a crossing, it should solve the path following problem for the SWLs, until at least one crossing is detected. The task can be achieved by using only control variable $v_{\theta}$ and setting constant $v_{x}=v_{f}>0$ and null $v_{y}=0$. Linear feedback control can be realized by tangent linearization of $\dot{b}$ and $\dot{\alpha}$, in the neighborhood of $(b=0, \alpha=0)$. This gives a second order linear system which is controllable, and thus asymptotically stabilizable by linear feedback on $v_{\vartheta}$ with an appropriate choice of positive gains $k_{2}$ and $k_{3}$ :

$$
v_{\vartheta}=\left(k_{2} b-k_{3} \alpha\right) v_{f}
$$

Plan path to destination When a crossing is detected with the CSE, and tracked with the VLT, the robot has univocally identified its $I D$ and orientation. This information, along with the CS position in the robot frame, and with the map, identifies the robot pose (position and orientation). The robot then utilizes a Dijkstra-based graph search to find the shortest path to the destination. Depending on the result of the graph search, the robot will approach and follow another street (repeat the corresponding actions in the plan), or stop if the crossing corresponds to the desired destination.

\section{Experiments}

In this section, we will outline the main characteristics of the ASPICE system, and show the results of two experiments: the first is a comparison between VBN and the two existing ASPICE navigation modes, while the second achieves autonomous battery charging (clips are available at: http://www. dis. uniromal. it/ labrob/research /ASPICE.html).

The ASPICE system enables users to drive several output devices (the domotic appliances or AIBO). The video feedback from the robot camera to the user is fundamental for increasing his/her sense of presence within the environment. Several input devices (including a Brain Computer Interface) can be used to select commands, for driving AIBO, through a Graphic User Interface (GUI). Since an objective of the project is compatibility with a variety of users and their level of disability, three navigation modes have been developed for AIBO: Single step, Semi-autonomous and Autonomous mode. Each navigation mode is associated to a ASPICE GUI (see Fig. 3). In single step mode, the robot can be driven, with a fixed step size, in any six directions (forward, backward, lateral left/right, $\mathrm{CW}$ and $\mathrm{CCW}$ rotations). Before performing the command, the robot verifies with the distance sensors if the step can be performed without colliding. In semi-autonomous mode, the user specifies the main direction of motion: the robot walks continuously in that direction until it receives a new command (either a new direction or a stop) and concurrently uses artificial potential fields, based on the distance 


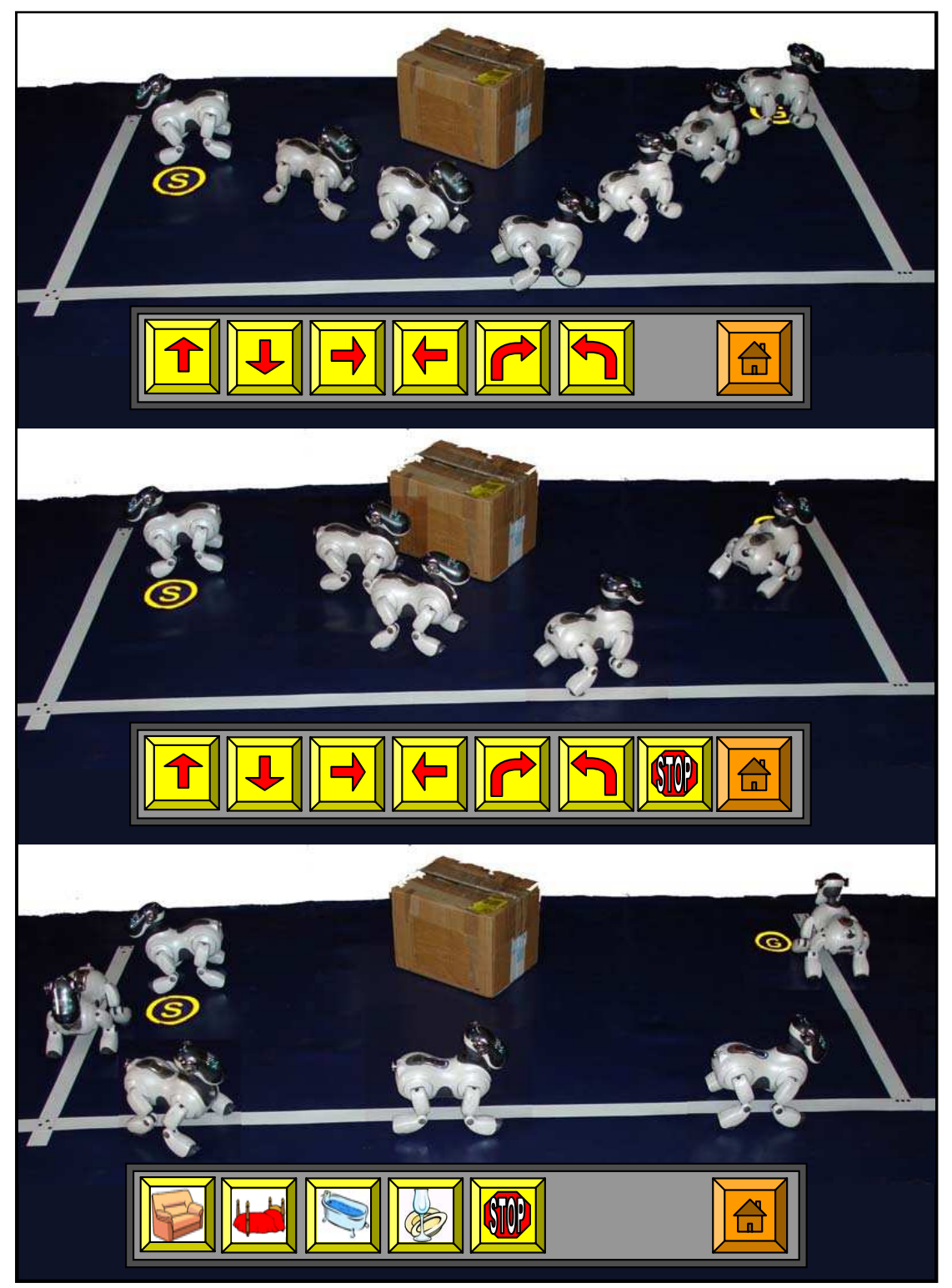

Fig. 3: Comparing navigation modes: AIBO is driven from $\mathrm{S}$ to $\mathrm{G}$ with single step (above), semiautonomous (center) and autonomous (below) modes. The ASPICE Navigation GUIs for each mode are also shown; in each GUI, the home button brings back to the ASPICE main GUI.

sensors, to avoid obstacles. Finally, in the autonomous mode, only a target point in the environment is assigned by the user, and the robot travels to the target (e.g., the living room, the bathroom, or the kitchen). This mode is useful for quickly reaching some 


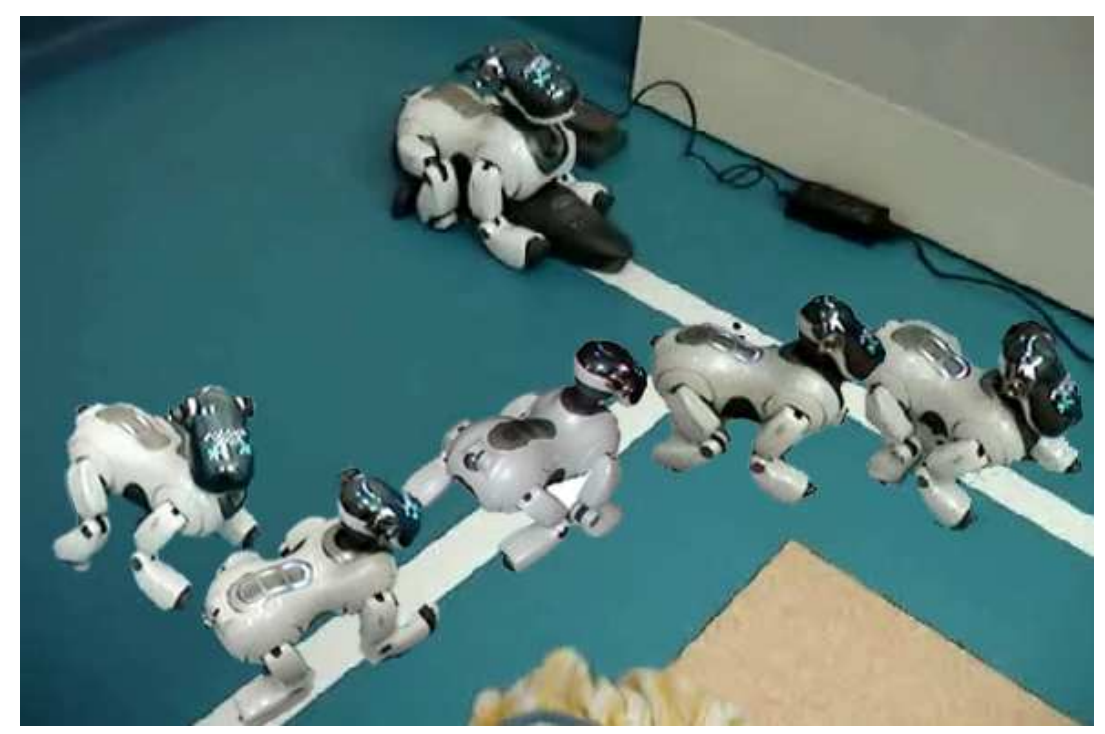

Fig. 4: The battery charging experiment.

Table 1: Comparison between the three ASPICE navigation modes.

\begin{tabular}{rcc}
\hline & execution time (sec) & user intervention (clicks) \\
\hline single step & 107 & 11 \\
semi-autonomous & 83 & 5 \\
autonomous & 90 & 1 \\
\hline
\end{tabular}

important locations; it is particularly useful for severely impaired patients, which are unable to send frequent commands. The practical implementation of the autonomous mode is the vision-based path planner/follower presented in this paper.

In a first experiment (Fig. 3), the user is expected to drive the robot, by selecting commands on the ASPICE GUIs, from a start point to a goal point (denoted respectively by "S" and "G" on the image). The task is repeated 5 times for each navigation mode (single step, semi-autonomous, and autonomous) and results are averaged. Comparison between the three modes is based on execution time and user intervention (i.e., number of times the user had to intervene by clicking on the GUI for updating the commands). As expected, results (indicated in Table 1) confirm the qualitative properties of the autonomous mode. Note, however, that the best choice depends not only on user's preference and ability but also on the specific task (e.g., position of the start and goal points in the environment, presence and position of obstacles).

In a second experiment, autonomous battery charging is tested. This experiment provides an additional testbed for VBN. In fact, the AIBO Charging Station is placed near a marked crossing on the roadmap, and as soon as the battery level is low, the robot 
autonomously moves to the station. The experiment is illustrated in Fig. 4. The robot position at consecutive time frames is shown while it approaches the roadmap, follows the street up to the battery charger crossing, detects it, and makes a turn in order to reach the charging station on the basis of the plan.

\section{Conclusions and future work}

A vision-based navigation system for AIBO has been developed. This system has been integrated in the ASPICE system, compared with the two previously designed ASPICE navigation modes, and tested by patients in a neurorehabilitation program. For two weekly sessions over 4 weeks, patients suffering from Spinal Muscular Atrophy type II and Duchenne Muscular Dystrophy have been practising with the ASPICE system. All of the patients were able to master the system and control AIBO within 5 sessions. The average grade given by the patients to their "personal satisfaction in utilizing the robot' was 3.04 on a 5-point scale [12]. This is a very promising result, considering that the users were originally more accustomed to using and controlling the 'traditional' ASPICE appliances rather than the mobile robot.

\section{References}

1. G. N. DeSouza, A. C. Kak: Vision for Mobile Robot Navigation: A Survey. In: IEEE Transactions on Pattern Analysis and Machine Intelligence Vol. 24, n. 2 (2002)

2. M. Carreras, P. Ridao, R. Garcia, T. Nicosevici: Vision-based localization of an underwater robot in a structured environment. In: IEEE International Conference on Robotics and Automation (2003)

3. M. Kabuka, A. Arenas: Position verification of a mobile robot using standard pattern. In: IEEE Journal of Robotics and Automation, Vol. 3 n. 6 (1987) 505 - 516

4. L. W. Ching, M. K. H. Leung: SINVI: smart indoor navigation for the visually impaired. In: 8th International Control, Automation, Robotics and Vision Conference, Vol. 2 (2004)

5. A. M. Sabatini, V. Genovese, E. S. Maini: Toward low-cost vision-based 2D localisation systems for applications in rehabilitation robotics. In: IEEE/RSJ International Conference on Intelligent Robots and System, Vol. 2 (2002) 1355 - 1360

6. F. Cincotti, F. Aloise, F. Babiloni, M. G. Marciani, D. Morelli, S. Paolucci, G. Oriolo, A. Cherubini, F. Sciarra, F. Mangiola, A. Melpignano, F. Davide, D. Mattia: Brain-operated assistive devices: the ASPICE project. In: 1st IEEE/RAS-EMBS International Conference on Biomedical Robotics and Biomechatronics (2006)

7. L. G. Roberts: Machine perception of three-dimensional solids. In: Optical and Electro-optical Information Processing, M I T Press, Cambridge, MA (1965)

8. T. Rofer and others: Robocup 2005 German Team Technical Report, Center for Computing Technology - Universität Bremen (2005)

9. S. Wesolkowski, M. E. Jernigan, R. D. Dony: Comparison of color image edge detectors in multiple color spaces. In: International Conference on Image Processing, Vol. 2 (2000)

10. P. I. Corke: Visual control of robots: high performance visual servoing, Research Studies Press LTD (1996)

11. V. A. Ziparo, L. Iocchi: Petri Net Plans. In: Fourth International Workshop on Modelling of Objects, Components, and Agents (MOCA'06), Turku, Finland (2006)

12. F. Cincotti, D. Mattia, F. Aloise, S. Bufalari, G. Schalk, G. Oriolo, A. Cherubini, M. G. Marciani, F. Babiloni: Non invasive Brain-Computer Interface systems: towards their application as assistive technology. Submitted to Brain Research Bulletin (2006) 